\title{
Implicando al alumnado para que sea consciente de su nivel de conocimientos
}

\section{Jorge Díez}

Departamento de Informática, Escuela Politécnica de Ingeniería de Gijón, Universidad de Oviedo. jdiez@,uniovi.es

\section{\$EWWFW}

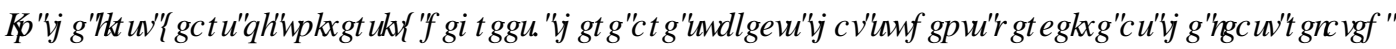
WRIUKHGH UHWKH QDUHWXG IQJ

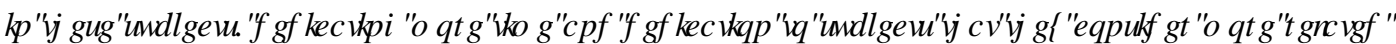

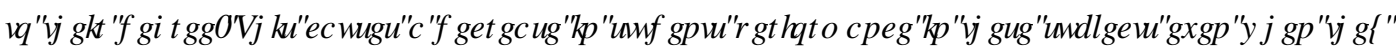

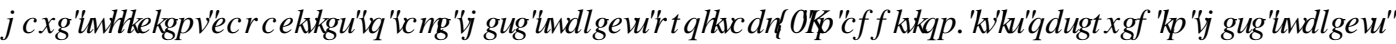

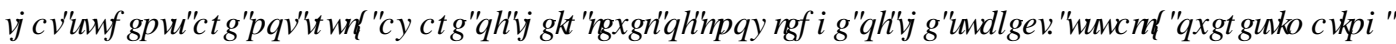

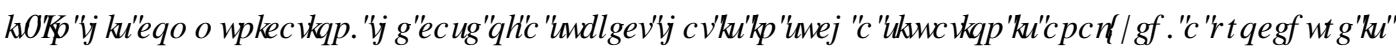

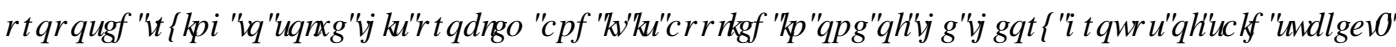

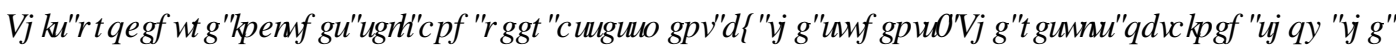
HIHFUYHQHMRIIUKHSLRSRUHCTSURFHCXUL

\section{. HZ RLGVIUHDDWHP HQWSHUDDMMP HQWIHLEDFN}

[

\section{HXP HQ}

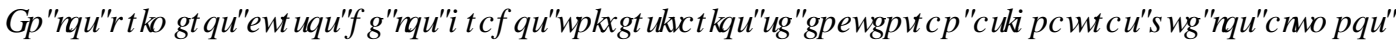

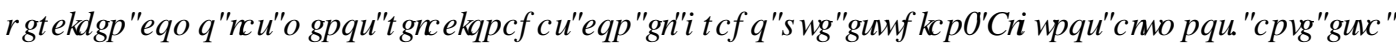

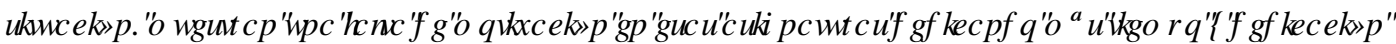

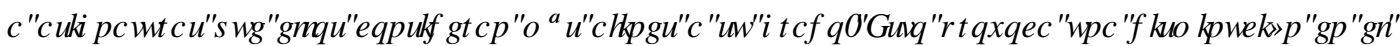

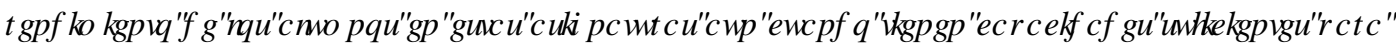

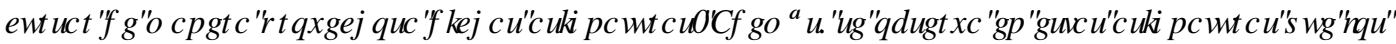

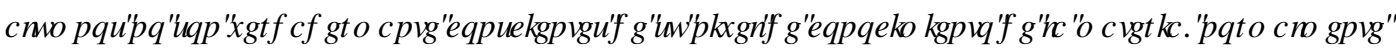

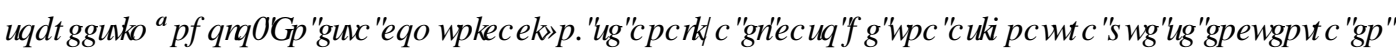

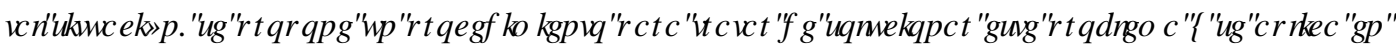

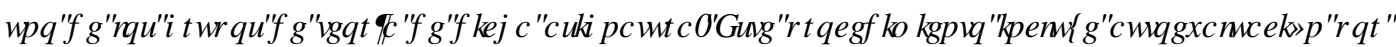

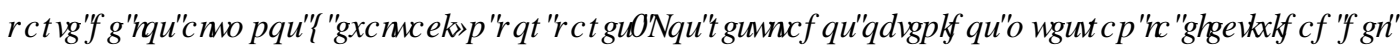
SLRFHUP IHQMRISLRSXHMRII

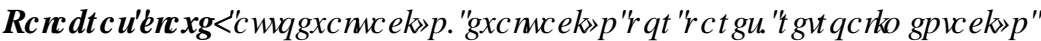

\section{Introducción}

A la hora de afrontar el aprendizaje de una nueva materia un factor importante para que el aprendizaje sea un éxito es la motivación del alumnado. Cuando hablamos de estudiantes universitarios es fácil que exista esa motivación ya que, normalmente, cursan grados en los que están interesados. Sin embargo, en su primer año en la universidad, los estudiantes deben cursar asignaturas que consideran, bajo su punto de vista, ajenas a sus intereses por no estar claramente identificadas con el grado que cursan. 
La falta de motivación de los alumnos por este tipo de asignaturas, que suelen presentarse más en los primeros cursos, provoca que muchos de ellos no presten la atención suficiente a las mismas a lo largo del semestre en el que se imparten (centrándose en otras que les resultan más afines). Después, cuando deben realizar alguna tarea propuesta en la evaluación continua de dichas asignaturas, tratan de ponerse al día, pero a veces ya es demasiado tarde y en lugar de aplicar los conocimientos adquiridos para resolver las tareas, la evaluación continua se convierte en una carrera de supervivencia. Lograr que los alumnos se impliquen más con estas asignaturas es una tarea ardua para la que no existe solución sencilla.

En este artículo se va a relatar la experiencia llevada a cabo en una asignatura de estas características. Se trata concretamente de la asignatura ) XQCDP HQURVGH, QRRP $i$ WFD, asignatura de 6 créditos ECTS de primer semestre y curso, que es común a todos los grados que se imparten en la Escuela Politécnica de Ingeniería de Gijón (Universidad de Oviedo). Esta escuela imparte un grado de informática, otro de telecomunicaciones y 6 grados de industriales, siendo el examen de esta asignatura común para todos los grados. Los alumnos del grado de informática se reparten en dos grupos de teoría, los de telecomunicaciones en otros dos grupos y los de industriales se distribuyen en 8 grupos de teoría.

La materia de la asignatura se ha elaborado para cubrir la competencia específica: "\&RQRFIP IHQURVEI UFRV

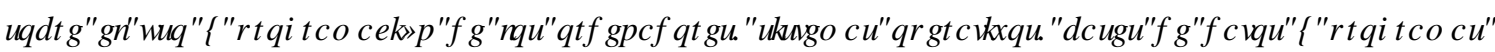

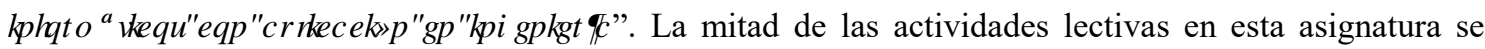
dedican a enseñar a los alumnos los fundamentos de la programación y muchos de ellos no se sienten motivados pues piensan que nunca tendrán que implementar ningún programa.

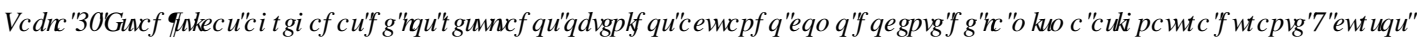

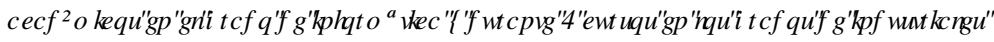

\begin{tabular}{ccccccc}
\hline Grado & $\begin{array}{c}\text { Cursos } \\
\text { académicos }\end{array}$ & $\begin{array}{c}\text { Número de } \\
\text { alumnos }\end{array}$ & $\begin{array}{c}\text { Número de } \\
\text { alumnos } \\
\text { por curso }\end{array}$ & $\begin{array}{c}\text { Alumnos } \\
\text { presentados } \\
\text { en enero }\end{array}$ & $\begin{array}{c}\text { Aprobados } \\
\text { sobre } \\
\text { presentados }^{2}\end{array}$ & $\begin{array}{c}\text { Aprobados } \\
\text { sobre } \\
\text { matriculados }^{3}\end{array}$ \\
\hline Informática & 5 & 310 & 62 & $79,50 \%$ & $65,02 \%$ & $51,31 \%$ \\
Industriales & 2 & 133 & 66,5 & $88,69 \%$ & $44,06 \%$ & $39,08 \%$ \\
\hline
\end{tabular}

El autor de este artículo ha sido profesor de esta asignatura durante varios cursos académicos. De 2011 a 2016 ha sido profesor de la asignatura en el grado de informática y de 2016 a 2018 en los grados de industriales (en uno de sus grupos de teoría). En la Tabla 1, se muestran ciertas estadísticas agregadas de los cursos mencionados en la convocatoria de enero, que es la convocatoria que se evalúa de manera continua. Como se puede apreciar, en los 5 cursos académicos impartidos en el grado de informática, el porcentaje de aprobados sobre el total de matriculados en la convocatoria de enero ha sido del 51,31\% $(65,02 \%$ sobre los presentados) y se han presentado el 79,50\% de los alumnos. En los 2 siguientes cursos académicos en los que se impartió la misma asignatura, con los mismos contenidos y el mismo mecanismo

\footnotetext{
${ }^{1}$ Es el equivalente a la Tasa de Evaluación aplicado a esta asignatura en la convocatoria de enero. Tasa de Evaluación: relación porcentual entre el número de créditos presentados a evaluación por los estudiantes matriculados en el curso académico referenciado y el número total de créditos matriculados. Los créditos reconocidos o transferidos no están incluidos ni en los créditos presentados ni en los matriculados.

${ }^{2}$ Se trata de un valor equivalente a la Tasa de Éxito, pero aplicada a esta asignatura sobre los resultados obtenidos en enero. Tasa de Éxito: relación porcentual entre el número de créditos superados por los estudiantes matriculados en el curso académico referenciado y el número total de créditos presentados a evaluación en dicho curso. Los créditos reconocidos o transferidos no están incluidos ni en los créditos superados ni en los presentados.

${ }^{3}$ Este valor es equivalente a la Tasa de Rendimiento para la convocatoria de enero en esta asignatura. Tasa de Rendimiento: relación porcentual entre el número de créditos superados por los estudiantes matriculados en el curso académico referenciado y el número total de créditos matriculados en dicho curso. Los créditos reconocidos o transferidos no están incluidos ni en los créditos superados ni en los matriculados.
} 
de evaluación pero en los grados de industriales, se presentaron a la convocatoria de enero el $88,69 \%$ de los alumnos superando la asignatura el $44,06 \%$ de los presentados lo que supone el $39,08 \%$ de los matriculados. Se puede apreciar claramente un peor rendimiento de los alumnos de los grados de industriales respecto a los alumnos del grado de informática. Esto puede parecer lógico, pero hay que tener en cuenta, como ya se ha comentado anteriormente, que la asignatura es de primer curso y semestre y no se precisan conocimientos más allá de los que se adquieren en bachillerato, que son bastante similares en todos los alumnos que acceden a cursar una ingeniería. Por tanto, la causa de esa diferencia en rendimiento parece deberse a una falta de motivación por parte de los alumnos de los grados de industriales, motivación, por otra parte, que sí parecen tener los alumnos del grado de informática.

Llama también la atención que el porcentaje de alumnos presentados en la convocatoria de enero es claramente superior en los grados de industriales respecto al de informática, lo cual hace pensar que los alumnos no son conscientes de su verdadero nivel de conocimiento de la materia y se presentan a la prueba teórica final sobreestimando, quizás, sus conocimientos.

Con objeto de hacer que los alumnos sean conscientes de su verdadero nivel de conocimientos de la materia y, a su vez, corregir la falta de motivación, se preparó una actividad que pudiese resultar atractiva para los alumnos, que les permitiese comparar sus conocimientos con los del resto de compañeros de forma anónima y que a la vez les motivase en la parte de la asignatura que más complicada les resulta: la parte de programación. La actividad se basa en el uso de dos mecanismos de evaluación diferentes: la autoevaluación y la evaluación por pares. Coll Aliaga y Martinez-LLario (2018) ya utilizaron ambos mecanismos de evaluación en una misma asignatura, aunque no para evaluar la misma actividad como ocurre en la experiencia presentada en este artículo. Existe una extensa literatura donde se enumeran las bondades de este tipo de correcciones en las que participan los alumnos (Falchikov, 2000; Panadero, 2017; Topping, 2003; Wanner, 2018). En ellas se discuten aspectos sobre el diseño de la metodología a utilizar y se indica que es necesario tener una buena planificación antes de ponerla en funcionamiento.

En la experiencia llevada a cabo, no se pretende cambiar la evaluación de la asignatura (los alumnos seguirán siendo evaluados por el profesor) sino que se pretende que los alumnos participen en la evaluación de un trabajo evaluándose a sí mismos y evaluando también a algunos de sus compañeros. Con esto se piensa que los alumnos serán conscientes de su conocimiento de la materia y les añadirá un extra de motivación.

\section{Objetivos}

En la experiencia que se ha llevado a cabo, se han marcado dos objetivos principales:

- conseguir que los alumnos tengan una visión crítica de sus conocimientos de la materia y

- motivar a los alumnos en la parte de la materia que les resulta más complicada, con lo que se logrará mejorar los conocimientos adquiridos por los alumnos y, por consiguiente, mejorar el rendimiento final de los alumnos en la asignatura.

Para lograr estos objetivos se plantea proponer a todos los alumnos una tarea individual que será evaluada por diferentes actores: i) el propio alumno (dos veces, una vez al principio y otra al final), ii) varios compañeros (actuando el propio alumno como evaluador del trabajo de otros compañeros) y iii) el profesor.

La manera de medir si la experiencia ha sido un éxito o no, se basará en comparar los resultados obtenidos por los alumnos en la convocatoria de enero respecto con los resultados registrados en cursos anteriores en la misma convocatoria.

(c)) BY-NC-ND 2021, Universitat Politècnica de València

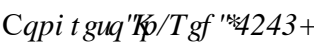




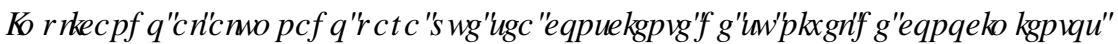

Para constatar en qué medida los alumnos han sido críticos con su propio trabajo se analizará la diferencia de puntuación obtenida por los alumnos en las dos evaluaciones que realizaron de su propio trabajo (una antes de ver los trabajos de sus compañeros y otra después). Se observará si ha habido mejora en el rendimiento analizando las tasas de éxito y de rendimiento.

\section{Desarrollo de la innovación}

Como ya se comentó en la Introducción, la experiencia se centra en los alumnos de la asignatura de Fundamentos de Informática de los grados de industriales en la Escuela Politécnica de Ingeniería de Gijón. La experiencia se llevó a cabo con los estudiantes de uno de los grupos en el curso 2019-2020. A ese grupo le fueron asignados 54 alumnos, todos ellos potenciales participantes en la experiencia. A todos se les ofreció la posibilidad de participar en la misma mostrándoles la mejora en la comprensión de los contenidos de la asignatura que podrían obtener y la posibilidad de compararse con sus compañeros de manera anónima. La participación fue voluntaria y sin incentivos, ya que no está claro cómo incluir incentivos sin alterar la evolución normal de la asignatura (Double, 2020). Participaron, finalmente, 37 alumnos en todas las correcciones solicitadas. En todo momento se insistió a los alumnos de la importancia de la experiencia en la que iban a participar tratando de mantenerlos motivados para que se esforzasen en realizar buenas correcciones, ya que si las correcciones se efectuasen con cierto desinterés la experiencia perdería su relevancia (Turner, 2011).

Una vez vista la mitad de la materia de programación, se planteó una actividad en la que los alumnos debían realizar tres pequeños programas utilizando las herramientas de programación habituales en la asignatura. Se les pidió que documentasen bien los programas con todos los comentarios que considerasen necesarios. La entrega de estos trabajos no se realizó enviando los archivos de texto que contenían los programas, sino que se les pidió a los alumnos que los enviasen como imágenes. Puede parecer extraño, pero el objeto de tal petición se debe a que, a la hora de corregir, se pretendía que los alumnos leyesen los programas y tratasen de comprenderlos. Se quería evitar a toda costa que los alumnos se limitasen a ejecutar los programas y ver simplemente si funcionaban.

Una vez que los trabajos se entregaron se les proporcionó a los alumnos una rúbrica en la que se aportaba una posible solución a los problemas planteados y además una serie de directrices acerca de cómo se deberían evaluar los trabajos. Posteriormente, se les pidió que evaluasen su propio trabajo siguiendo dichas directrices.

Tras esta primera fase de autoevaluación, se le entregaron a cada alumno los trabajos de 3 compañeros completamente anonimizados y se les pidió que evaluasen dichos trabajos siguiendo la rúbrica que se les había proporcionado. Al finalizar esta segunda fase los alumnos sólo eran conocedores de la nota que habían puesto a su propio trabajo y las notas que había otorgado a los trabajos que les había tocado evaluar.

Con la experiencia adquirida tras las correcciones efectuadas en la primera y segunda fases, se les pidió a los alumnos que volviesen a evaluar su propio trabajo. En esta tercera fase, es de suponer que tras haber visto otros trabajos y al haber adquirido cierta experiencia en la corrección, los alumnos cambien la calificación que habían otorgado a sus trabajos en un primer momento.

Para finalizar, se publicó una lista (anonimizada) con las calificaciones de cada trabajo: i) la otorgada por el profesor y ii) las tres obtenidas por los alumnos en el proceso de evaluación por pares. Con esta publicación se perseguía que los alumnos viesen cómo habían valorado su trabajo los compañeros y cómo lo había hecho el profesor. Las calificaciones de la autoevaluación no se hicieron públicas y cada alumno sólo conocías las suyas. 


\section{Resultados}

En la Tabla 2, se muestra el rendimiento obtenido en la convocatoria de enero tras aplicar la innovación descrita en el apartado anterior. Se puede apreciar que el porcentaje de alumnos presentados en esa convocatoria se redujo ligeramente y el porcentaje de aprobados sobre presentados y sobre matriculados se incrementó significativamente. Por tanto, parece que la innovación sí que influyó en los alumnos de la manera deseada.

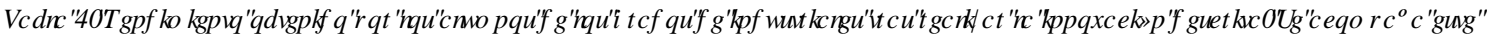

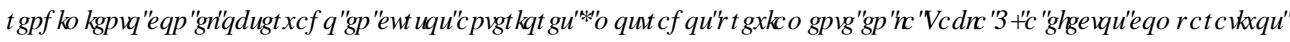

\begin{tabular}{ccccccc}
\hline Grado & $\begin{array}{c}\text { Cursos } \\
\text { académicos }\end{array}$ & $\begin{array}{c}\text { Número de } \\
\text { alumnos }\end{array}$ & $\begin{array}{c}\text { Número de } \\
\text { alumnos } \\
\text { por curso }\end{array}$ & $\begin{array}{c}\text { Alumnos } \\
\text { presentados } \\
\text { en enero }\end{array}$ & $\begin{array}{c}\text { Aprobados } \\
\text { sobre } \\
\text { presentados }\end{array}$ & $\begin{array}{c}\text { Aprobados } \\
\text { sobre } \\
\text { matriculados }\end{array}$ \\
\hline Informática & 5 & 310 & 62 & $79,50 \%$ & $65,02 \%$ & $51,31 \%$ \\
Industriales & 2 & 133 & 66,5 & $88,69 \%$ & $44,06 \%$ & $39,08 \%$ \\
\hline $\begin{array}{c}\text { Industriales } \\
\text { tras la } \\
\text { experiencia }\end{array}$ & $\mathbf{1}$ & $\mathbf{5 4}$ & $\mathbf{5 4}$ & $\mathbf{8 5 , 1 8 \%}$ & $\mathbf{6 0 , 8 7 \%}$ & $\mathbf{5 1 , 8 5 \%}$ \\
\hline
\end{tabular}

En la Figura 1 se muestra la variación de notas entre las dos autoevaluaciones (eje y) frente a la nota media obtenida en la evaluación por pares (eje x). A modo de ejemplo, se puede apreciar a un alumno que fue calificado con un 1.1 en la evaluación por pares y que en su segunda autoevaluación redujo su puntuación en algo más de 3 puntos. En el extremo opuesto se puede ver a un alumno que fue calificado por sus compañeros con un 8.8 y que al reevaluarse incrementó su calificación en 2.3 puntos. Evidentemente, no todos los alumnos presentan esta diferencia tan marcada. Se representa también en la figura la línea de tendencia con su ecuación, que tiene una pendiente positiva. Si se entra un poco más en detalle, se encuentran resultados interesantes. El 70,27\% de los alumnos (26 de 37) ha rebajado su calificación en la segunda evaluación de su propia tarea, el 10.81\% (4 alumnos) han considerado que su nota se mantenía y el $18.92 \%$ restante ( 7 alumnos) consideraron que merecían una calificación mejor.

Por tanto, los resultados parecen reflejar que, en general, los alumnos analizaron de forma más crítica su propio trabajo en la segunda evaluación después de haber visto y evaluado los trabajos de otros compañeros. Parece que examinar en detalle otros trabajos para evaluarlos ha llevado a los alumnos a encontrar defectos que antes no habían detectado en su entrega. Es importante recordar que los alumnos no conocían las notas que les habían puesto sus compañeros en el momento de revisar su propio trabajo por segunda vez. Esta visión más crítica de su nivel de conocimiento de la materia puede haber tenido influencia en el decremento del porcentaje de alumnos presentados en enero que se aprecia en la Tabla 2.

Por otra parte, la tendencia mostrada en la Figura 1 parece querer decir que los alumnos con trabajos peores rebajaron su nota en gran medida en la segunda autoevaluación mientras que los alumnos con mejores trabajos redujeron menos su calificación o incluso la mejoraron en algunos casos. 


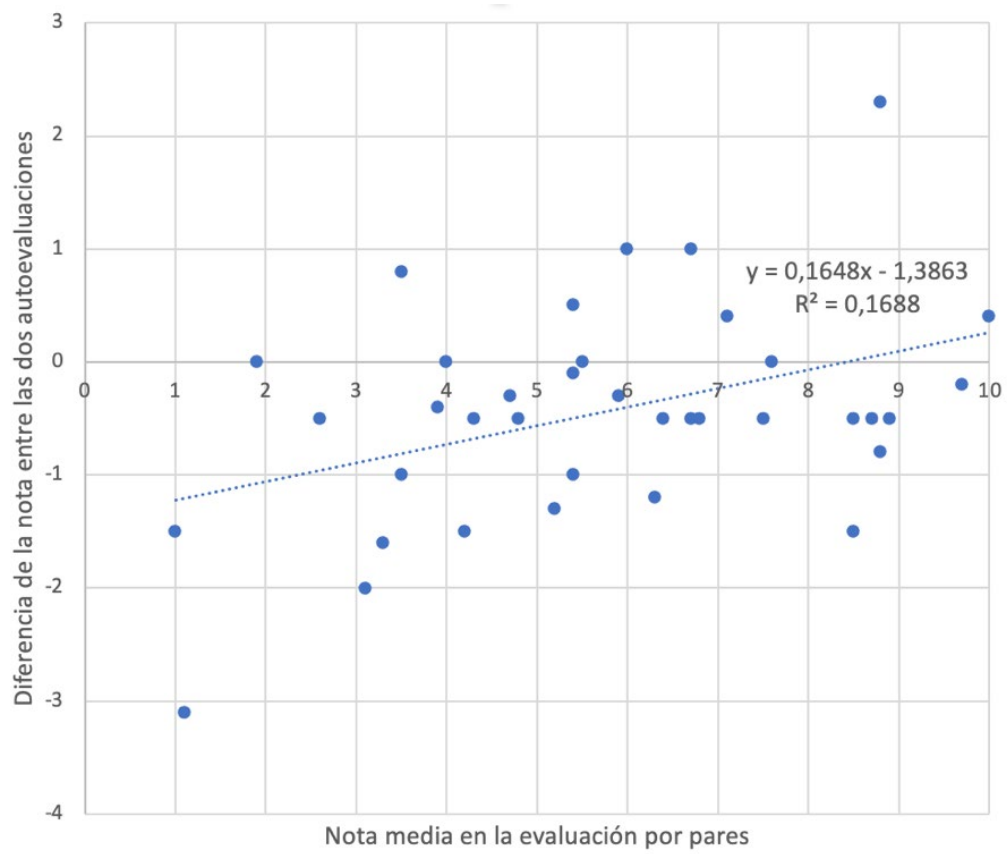

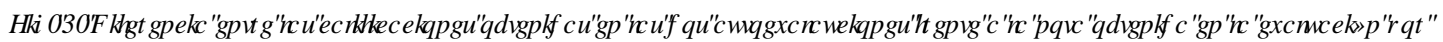

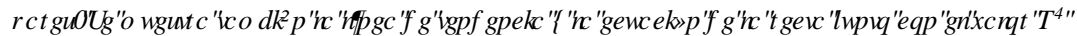

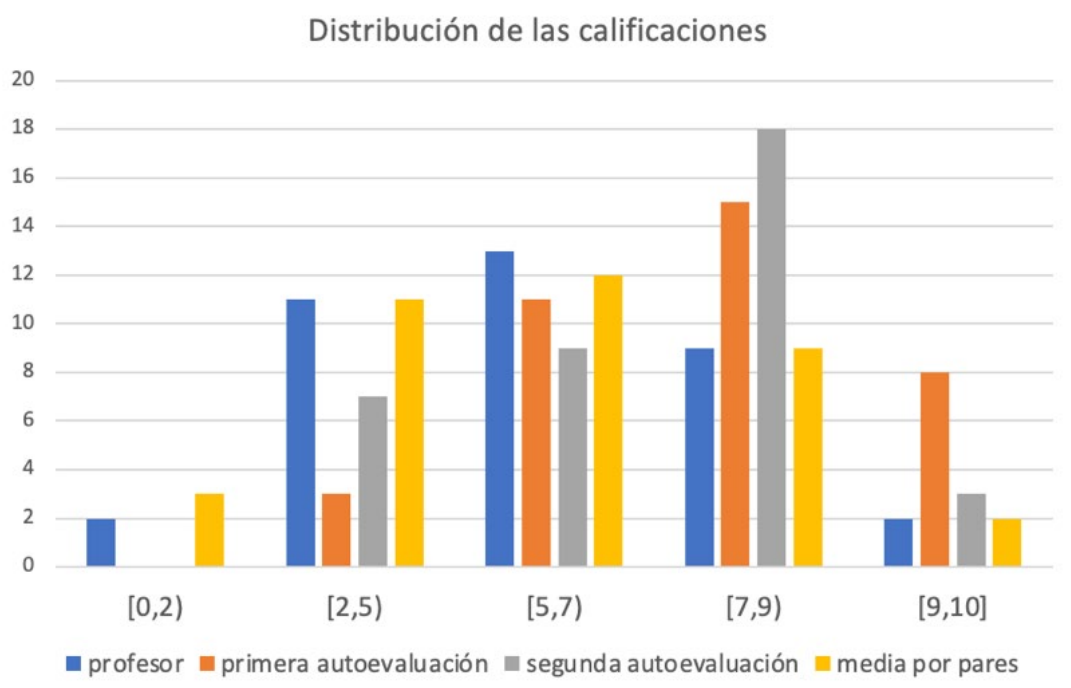

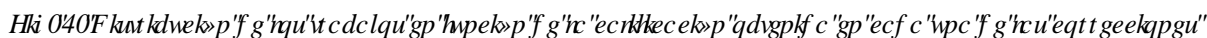

Puesto que se cuenta con 4 calificaciones para cada trabajo (profesor, dos autoevaluaciones y evaluación por pares), se han comparado dichas calificaciones en términos agregados. En la Figura 2 se puede observar la distribución de las calificaciones obtenidas por los trabajos en las 4 correcciones efectuadas. Se aprecia que los alumnos, cuando evaluaron su propio trabajo por primera vez, se calificaron mayoritariamente con notas a partir del 5. Sin embargo, en la segunda autoevaluación se puede apreciar cómo aumenta el número de suspensos. Otro aspecto interesante que se puede observar es que la distribución de las calificaciones obtenidas mediante la evaluación por pares es muy similar a la obtenida por las calificaciones del profesor. 
$\left.-R U H^{\prime} t H\right]$

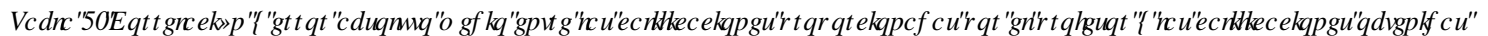

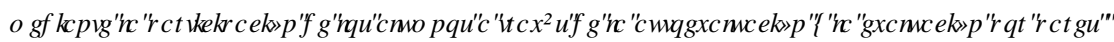

\begin{tabular}{ccc}
\hline Grado & Correlación & $\begin{array}{c}\text { Error Absoluto } \\
\text { Medio (EAM) }\end{array}$ \\
\hline Primera autoevaluación & 0,6450 & 2,05 \\
Segunda autoevaluación & 0,8175 & 1,56 \\
$\begin{array}{c}\text { Media en la evaluación } \\
\text { por pares }\end{array}$ & 0,7887 & 1,37 \\
\hline
\end{tabular}

Para analizar en qué medida se asemejan las calificaciones otorgadas por el profesor con respecto al resto de calificaciones obtenidas con la intervención de los alumnos, se han calculado las correlaciones y el error absoluto medio (en adelante EAM) (Kotz, 1982), que es la media de las diferencias entre las calificaciones en valor absoluto. En la Tabla 3 se pueden ver los resultados obtenidos. Fijándose en la correlación, se ve que la correlación entre las calificaciones del profesor y las de los alumnos en la primera autoevaluación es la más baja. Se incrementa bastante en la segunda autoevaluación, lo que corrobora los análisis expuestos anteriormente acerca de que los alumnos revisaron sus trabajos de manera más crítica la segunda vez. La segunda autoevaluación y la evaluación por pares presentan correlaciones similares. En cuanto al EAM, los alumnos se otorgaron inicialmente calificaciones que distaban en media 2.05 puntos de la calificación otorgada por el profesor. En la segunda autoevaluación, la media de las diferencias en la calificación se redujo a 1.56, mostrando nuevamente que el alumno es más consciente de su conocimiento de la materia. En el caso de la evaluación por pares, el EAM es 1.37, lo que implica que la nota obtenida por este medio es en media 1.37 puntos mayor o menor que la otorgada por el profesor. Por tanto, aunque en la Figura 2 se veía que las calificaciones del profesor y de la evaluación por pares presentaban distribuciones similares, el EAM muestra que las calificaciones obtenidas mediante la revisión por pares en este experimento no son demasiado precisas. Una manera de mejorar estas calificaciones podría ser pedir a los alumnos que evalúen un número mayor de trabajos, de tal manera que la media fuese más significativa, sin embargo, esto cargaría con más trabajo a los alumnos y no parece que sea una solución práctica.

\section{Conclusiones}

Lo primero que hay que decir es que se debe ser cauto con las conclusiones puesto que el número de alumnos participantes no ha sido muy elevado y, también, porque los resultados presentados hacen referencia a un único curso académico.

De los resultados obtenidos, se puede concluir que la innovación descrita ha provocado una mejora en el rendimiento de los alumnos de los grados de industriales en la asignatura analizada. La mejora no ha supuesto obtener resultados equiparables a los obtenidos en el grado de informática en los 5 cursos analizados, pero sí ha supuesto una mejora respecto a lo visto en los 2 cursos académicos analizados con anterioridad de los grados de industriales.

También se puede concluir que los alumnos son más críticos con su nivel de conocimiento en la asignatura y así se ha visto reflejado en los resultados puesto que el porcentaje de alumnos presentados se ha reducido mientras que el de aprobados ha aumentado, claro indicador de que aumentó el número de alumnos que dejaron de presentarse al ser conscientes de que no estaban preparados. 
Finalmente, hay que decir que esta innovación podría incorporarse en la asignatura vistos los resultados. Sin embargo, no se debe abusar de este tipo de metodologías, ya que la carga de trabajo que ha supuesto tanto para el alumnado como para el profesorado ha sido elevada (Hanrahan, 2001).

\section{Referencias}

DOUBLE, K.S., MCGRANE, J.A. y HOPFENBECK, T.N. (2020). "The Impact of Peer Assessment on Academic Performance: A Meta-analysis of Control Group Studies" HQ( GXFDMRQDOB V FKRQRJ $55 H I I H$, vol. 32, p. 481-509.

KOTZ, S., JOHNSON, H.L. y READ, C.B. (1982). ( QF FCRSHGDRILWUUWHFDONFHQFH. New York.

COLL ALIAGA, E. y MARTINEZ-LLARIO, J.C. (2018). "La coevaluación y autoevaluación en la asignatura de Distribución de la Información Espacial del MUIGG en la UPV”. Garrigós Simón et al. (coord.). En ,112 ' 2 \&7पिएवा

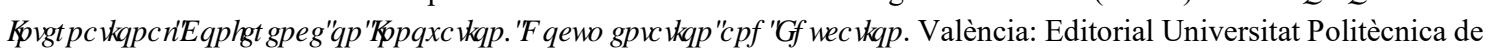
València. 637-645.

HANRAHAN, S.J. e ISAACS, G. (2001). "Assessing self-and peer-assessment: The students' views" HQ + IJKH】 ( GXFDMRQ5 HHDUFWD I' HIHRSP HQWvol. 20, issue 1, p. 53-70.

FALCHIKOV, N. y GOLDFINCH, J. (2000). "Student peer assessment in higher education: A meta-analysis comparing peer and teacher marks" HQ5 HIHELRIC GXFDWRQDO5 HMDUFK, vol. 70, issue 3, p. 287-322.

PANADERO, E. y BROWN, G.T.L. (2017). “Teachers' reasons for using peer assessment: positive experience predicts use" HQ( XURSHDQ-RXLQDORII3 V FKRQDJ IRIC GXFDMRQ vol. 32, issue 1, p. 133-156.

TOPPING, K. (2003). "Self and peer assessment in school and university: Reliability, validity and utility" en Segers

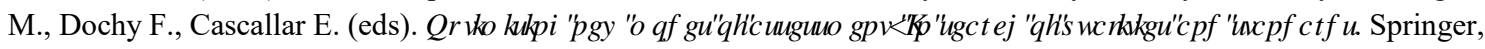
Dordrecht, p. 55-87.

TURNER, S.A., PÉREZ-QUIÑONES, M.A., EDWARDS, S.H. y CHASE, J.. (2011). "Student attitudes and

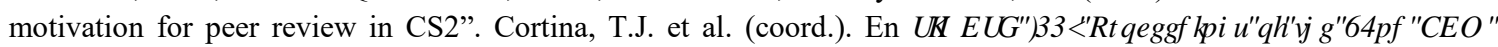
WFKQIFDON P SRUXP $\square R Q \& R P$ SXWAUTFHQFHHCXFDMRQ Dallas: Association for Computing Machinery. 347-352.

WANNER, T. y PALMER, E. (2018). "Formative self-and peer assessment for improved student learning: the crucial

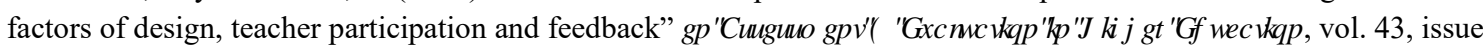
7, p. 1032-1047. 